\title{
MicroRNA 196B regulates FAS-mediated apoptosis in colorectal cancer cells
}

\author{
Ji-Su Mo ${ }^{1, *}$, Khondoker Jahengir Alam ${ }^{1, *}$, In-Hong Kang ${ }^{1}$, Won Cheol Park ${ }^{2}$, Geom- \\ Seog Seo ${ }^{3}$, Suck-Chei Choi ${ }^{3}$, Hun-Soo Kim¹, Hyung-Bae Moon ${ }^{1}$, Ki-Jung Yun ${ }^{1,3}$ and \\ Soo-Cheon Chae ${ }^{1,3}$ \\ ${ }^{1}$ Department of Pathology, School of Medicine, Wonkwang University, Iksan, Chonbuk, Republic of Korea \\ 2 Department of Surgery, School of Medicine, Wonkwang University, Iksan, Chonbuk, Republic of Korea \\ ${ }^{3}$ Digestive Disease Research Institute, Wonkwang University, Iksan, Chonbuk, Republic of Korea \\ * These authors contributed equally to this work
}

Correspondence to: Soo-Cheon Chae, email: chaesc@wku.ac.kr

Keywords: Fas, MIR196B, Colorectal cancer, Apoptosis

Received: August 11,2014 Accepted: December 22, $2014 \quad$ Published: December 26, 2014

This is an open-access article distributed under the terms of the Creative Commons Attribution License, which permits unrestricted use, distribution, and reproduction in any medium, provided the original author and source are credited.

\section{ABSTRACT}

Using miRNA microarray analysis, we identified 31 miRNAs that were significantly up-regulated or down-regulated in colon cancer tissues. We chose MIR196B, which was specifically up-regulated in colon cancer, for further study. We identified 18 putative MIR196B target genes by comparing between the mRNAs down-regulated in MIR196B-overexpressed cells and the assumed MIR196B target genes predicted by public bioinformatics tools. The association between MIR196B and FAS was verified in this study. FAS expression was constitutively elevated in normal human colorectal tissues. However, its expression was often reduced in human colorectal cancer. The decrease in FAS expression could be responsible for the reduction of apoptosis in colorectal cancer cells. In colorectal cancer tissue, we showed that MIR196B up-regulation was mutually followed by down regulation of FAS expression. We also showed that MIR196B directly repressed FAS expression in colorectal cells. Furthermore, anti-MIR196B up-regulated FAS expression and increased apoptosis in colorectal cancer cell lines. Our results suggest that the up-regulation of MIR196B modulates apoptosis in colorectal cancer cells by partially repressing FAS expression and that anti-MIR196B could be a potential candidate as an anti-cancer drug in colorectal cancer therapy.

\section{INTRODUCTION}

Colorectal cancer (CRC) is one of the most common cancers worldwide [1], and the third leading cause of cancer-related mortality in the United States [2]. Its etiology is very complex and not fully understood. Numerous epidemiological and biological studies have suggested that various risk factors, including nutrition, physical inactivity, obesity, and diabetes, play a critical role in the etiology of CRC [3, 4]. Most sporadic colorectal cancers are associated with inflammation and inflammatory diseases, such as inflammatory bowel disease (IBD) [5]. Several genetic factors, including microRNAs (miRNAs), are also thought to contribute to colorectal cancer risk. Much evidence suggests that miRNAs are important regulators of oncogenesis $[6,7]$. miRNAs are endogenously synthesized, short, noncoding RNA molecules of approximately 19-24 nucleotides. miRNAs contribute to the post-transcriptional regulation of gene expression in multicellular organisms by controlling the stability and translation of target mRNAs [8]. miRNAs contribute to the regulation of crucial biological processes, such as cell proliferation, apoptosis, differentiation, and angiogenesis [9]. They are also implicated in the pathogenesis of various diseases as tumor suppressor genes or oncogenes $[10,11]$. Therefore, characterization of miRNA expression patterns in cancer cells may have substantial value for disease diagnosis, prognosis, and therapy [12-14]. 
The members of the MIR196 gene family (MIR196A1, MIR196A2, and MIR196B) are transcribed from three different genes, which are located in homeobox (HOX) gene cluster regions in humans $[15,16]$. The MIR196A1 gene is located on Chr. 17q21.32 between the $H O X B 9$ and $H O X B 10$ genes. The MIR $196 A 2$ gene is located between $\mathrm{HOXC10}$ and $\mathrm{HOXC} 9$ on Chr. 12q13.13. The gene for MIR196B is located in an evolutionarily conserved region between $H O X A 9$ and HOXA10 on Chr. 7p15.2. The mature nucleotide sequences of MIR196A1 and MIR196A2 are identical, whereas mature MIR196B differs from MIR196A by one nucleotide [16]. Previous studies suggests that MIR196 may play critical roles in normal development and cancer pathogenesis by targeting specific genes [17].

In this study, we measured miRNA expression in colon cancer tissues and normal colon tissues by miRNA microarray analysis. We detected 31 microRNAs that were specifically up-regulated or down-regulated in colorectal cancer tissues. Of them, MIR196B was chosen for detailed analysis and further study. mRNA microarray expression profiles of MIR196B-overexpressing colorectal cancer cell lines were generated to identify MIR196B target molecules. The list of MIR196B target genes was narrowed down by comparison with a database of candidate target genes predicted by bioinformatics programs. We identified FAS cell surface death receptor (FAS, also called Apol or CD95) as a MIR196B target gene in colorectal cancer and verified their association between MIR196B and FAS in colorectal cancer cells.

\section{RESULTS}

\section{miRNA expression profiling in colon cancer tissue}

We used miRNA microarray analysis to compare the expression of miRNA precursors in colon cancer tissue and normal colon tissue. Total RNA for miRNA chip analysis was obtained from four different patients with colon cancer. We initially compared miRNA expression in normal colon tissues and the matched colon cancer tissues in two independent experiments. We identified 31 miRNAs that were up-regulated or down-regulated more than two-fold in both independent experiments (Table 1). The results from the miRNA chip analysis were validated by qRT-PCR for 11 miRNAs (Fig. 1A). We selected one, MIR196B, which was up-regulated in human colorectal cancer tissue, for further investigation in this study (Fig. 1B).

\section{mRNA expression profiling in MIR196B- overexpressing cells}

To determine the levels of endogenous MIR196B in SW480, Caco2, and HT29 cells, we carried out qRTPCR analysis using the total RNA isolated from the three different cell lines. As shown in Fig. 1C, the MIR196B level was lowest in Caco2 cells and highest in HT29 cells (Fig. 1C). To identify genes down-regulated by MIR196B overexpression, pre-MIR196B was transfected into SW480 and Caco2 cells. Increased expression of the miRNA $24 \mathrm{~h}$ after transfection in SW480 and Caco2 cells confirmed the transfection efficiency (Fig. S1A). Cells were harvested $48 \mathrm{~h}$ after transfection for mRNA expression profiling analysis with the Illumina HumanHT-12 v4 Expression BeadChip. We identified 67 genes whose expression was down-regulated 1.5 -fold in cells overexpressing MIR196B (Table S1).

\section{MIR196B target gene identification}

To identify MIR196B target genes, we compared the 67 candidate target genes identified by mRNA microarray analysis with candidate MIR196B target genes predicted by the TargetScan and miRWalk algorithms. Of the 67 genes, 18 were identified as putative targets of MIR196B (Table 2). Among them, we focused on FAS gene. To determine whether the expression levels of FAS in the colon cancer cell lines are comparable to the levels of MIR-196B, we carried out qRT-PCR or western blot analysis using the total RNAs or proteins isolated from SW480 and HT29 cells. HT29 cells show low FAS mRNA (Fig. S1B) and FAS (Fig. S1C) expression level compare to SW480 cells as MIR196B expression relatively higher in HT29 than SW480 cells. So, FAS expression might be depend on endogenous MIR196B expression in colon cancer cell lines.

\section{FAS is a target of MIR196B}

We investigated whether MIR196B regulated FAS mRNA and protein levels in SW480 cells. The FAS mRNA level was lower ( 0.66 fold) in SW480 cells transfected with pre-MIR196B than in un-transfected control cells (Fig. 2A). FAS protein expression was also downregulated (0.35 fold) in MIR196B-overexpressing cells (Fig. 2B).

To demonstrate a direct interaction between the FAS 3' UTR region and MIR196B, we cloned the WT FAS 3' UTR region predicted to interact with MIR196B into a luciferase vector (Fig. 2D). Luciferase activity decreased by $\sim 30 \%$ when cells were co-transfected with pre-MIR196B (Fig. 2E). As a control experiment, we cloned a mutated FAS 3' UTR sequence lacking ten 
Table 1: miRNAs differentially expressed in normal tissues and colon cancer tissues, as determined by miRNA microarray analysis

\begin{tabular}{|c|c|c|}
\hline miRNA & Chromosomal location & Fold change \\
\hline has-miR-7 & $9 \mathrm{q} 21.32$ & 6.58 \\
\hline hsa-miR-9-2 & $5 q 14.3$ & 4.68 \\
\hline has-miR-15a & $13 q 14.2$ & 2.91 \\
\hline has-miR-17 & $13 \mathrm{q} 31.3$ & 6.87 \\
\hline has-miR-19a & $13 \mathrm{q} 31.3$ & 4.17 \\
\hline has-miR-19b & $13 \mathrm{q} 31.3$ & 4.14 \\
\hline has-miR-20a & $13 \mathrm{q} 31.3$ & 7.05 \\
\hline has-miR-20b & $\mathrm{Xq} 26.2$ & 6.50 \\
\hline has-miR-21 & $17 \mathrm{q} 23.1$ & 3.60 \\
\hline has-miR-25 & $7 \mathrm{q} 22.1$ & 2.74 \\
\hline has-miR-27a & $19 \mathrm{p} 13.13$ & 2.78 \\
\hline has-miR-29a & $7 \mathrm{q} 32.3$ & 3.21 \\
\hline has-miR-29b & $7 \mathrm{q} 32.3$ & 5.49 \\
\hline has-miR-34a & $1 \mathrm{p} 36.22$ & 3.51 \\
\hline has-miR-34b & $11 \mathrm{q} 23.1$ & 2.34 \\
\hline hsa-miR-93 & $7 \mathrm{q} 22.1$ & 3.14 \\
\hline has-miR-106a & $\mathrm{Xq} 26.2$ & 5.55 \\
\hline has-miR-106b & $7 q 22.1$ & 3.49 \\
\hline has-miR-146a & $5 \mathrm{p} 34$ & 3.54 \\
\hline has-miR-139 & $11 \mathrm{q} 13.4$ & 0.53 \\
\hline has-miR-181c & $19 \mathrm{p} 13.13$ & 2.99 \\
\hline has-miR-196b & $7 \mathrm{p} 15.2$ & 7.18 \\
\hline has-miR-224 & $\mathrm{Xq} 28$ & 11.34 \\
\hline has-miR-374 & $\mathrm{Xq13.2}$ & 2.88 \\
\hline hsa-miR-375 & $2 \mathrm{q} 35$ & 0.33 \\
\hline has-miR-424 & $\mathrm{Xq} 26.3$ & 10.61 \\
\hline has-miR-452 & $\mathrm{Xq} 28$ & 3.42 \\
\hline has-miR-590 & $7 \mathrm{q} 11.23$ & 3.49 \\
\hline has-miR-622 & $13 \mathrm{q} 31.3$ & 2.56 \\
\hline has-miR-630 & $15 \mathrm{q} 24.1$ & 2.94 \\
\hline has-miR-765 & $1 \mathrm{q} 23.1$ & 2.49 \\
\hline
\end{tabular}

Four colon cancer tissue samples and matched normal colon tissue samples were analyzed twice with miRNA microarray. All differentially expressed miRNA had a $q$ value $<0.01$ (false-positive rate). All, except hsamiR-139 and has-miR-375, were up-regulated in colon cancer tissues.

of the complementary bases. As expected, repression of luciferase activity was abolished when the interaction between MIR196B and its target 3' UTR was disrupted (Fig. 2E). As additional control experiments, MIR1 instead of MIR196B was co-transfected with the WT and MT FAS 3' UTR constructs. Transfection of pre-MIR1 did not affect the luciferase activity of either construct (Fig. 2E). These results suggest that MIR196B directly regulates FAS expression in colorectal cancer cells.

\section{FAS gene expression in human colorectal cancer}

Given the findings described above, we investigated FAS expression in human colon cancer tissues and normal colon tissues by western blot analysis. FAS expression was down-regulated in all colon cancer tissues when compared to expression in normal colon tissues (Fig. 2C).

\section{MIR196B down-regulates FAS-mediated caspases in $\mathrm{SW480}$ cells}

To further define the functional interaction between MIR196B and FAS in SW480 cells, we investigated the expression of proteins involved in the FAS-mediated apoptotic pathway, such as active (cleaved) caspase 8 (CASP8) active (cleaved) caspase 3 (CASP3), as well as the expression of intrinsic apoptosis molecule active (cleaved) caspase 9 (CASP9) by western blot analysis. 
FAS (0.7 fold), CASP8 (0.69 fold), and CASP3 (0.71 fold) expression was markedly down-regulated by MIR196B transfection (Fig. 3A). These results indicate that MIR196B regulates FAS-mediated apoptosis by directly down-regulating FAS in colorectal cancer cells. We further investigated the expression of FAS, CASP8, CASP3, and CASP9 by transfection of anti-MIR196B into SW480 cells (Fig. 3A). FAS and CASP3 expression did not change after anti-MIR196B transfection. This was expected because the level of endogenous MIR196B in SW480 cells was very low, as shown in Fig. 1C. However, anti-MIR196B transfection increased CASP8 (1.19 fold) expression slightly. We also investigated the expression of FasL (also called FASLG or CD95L) in MIR196B- or
anti-MIR196B-transfected SW480 cells (Fig. 3A). FasL expression in SW480 cells was markedly down-regulated (0.7 fold) by MIR196B overexpression. However, there was no statistically significant change in FasL expression after anti-MIR196B transfection in SW480 cells. Interestingly, the FasL expression pattern was similar to the FAS expression pattern. These results suggest that MIR196B also regulates FasL expression, although we did not establish this in the present study.

\section{MIR196B directly regulates FAS expression}

To further investigate the down-regulation of FAS by MIR196B, we assessed the expression of FAS and

\section{A}

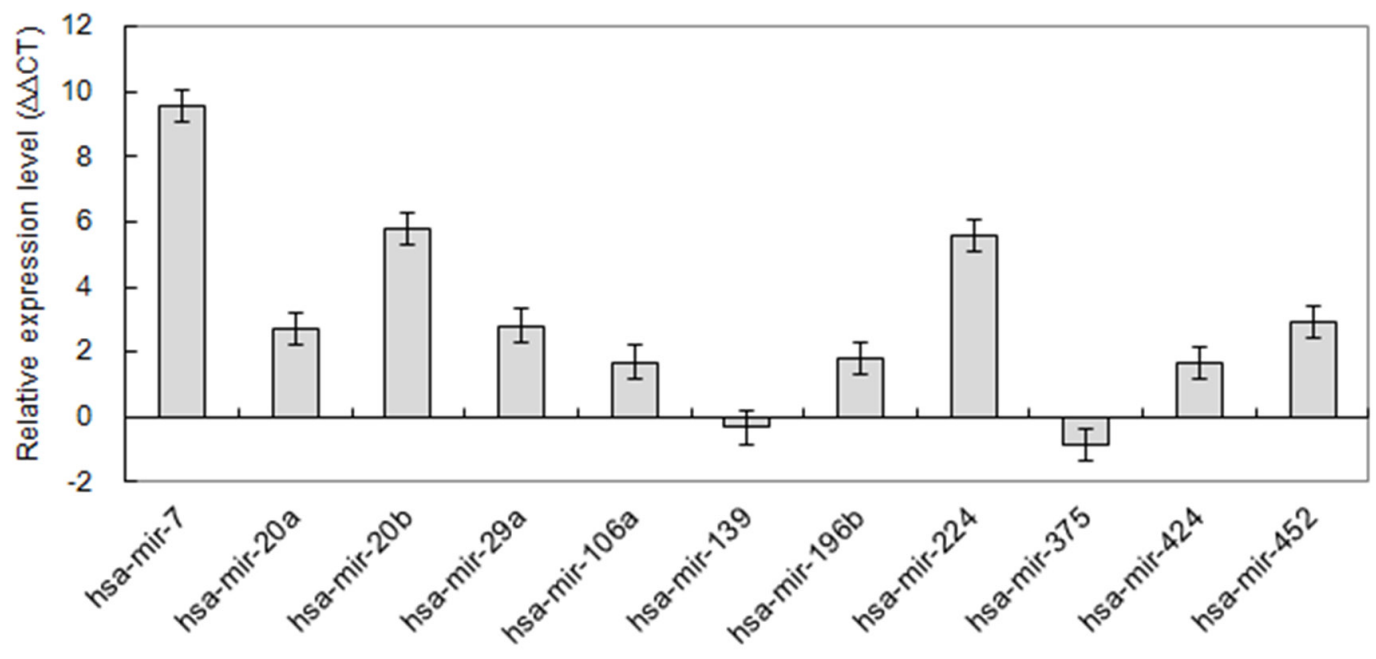

B

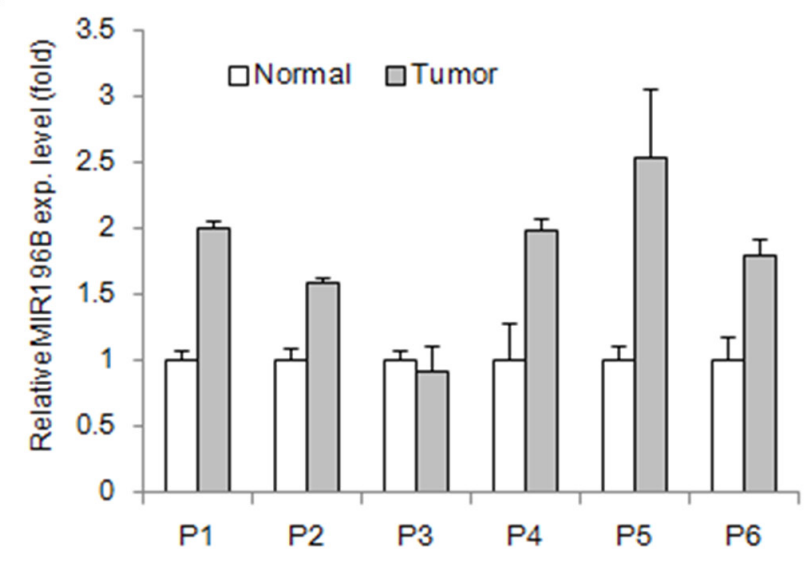

C

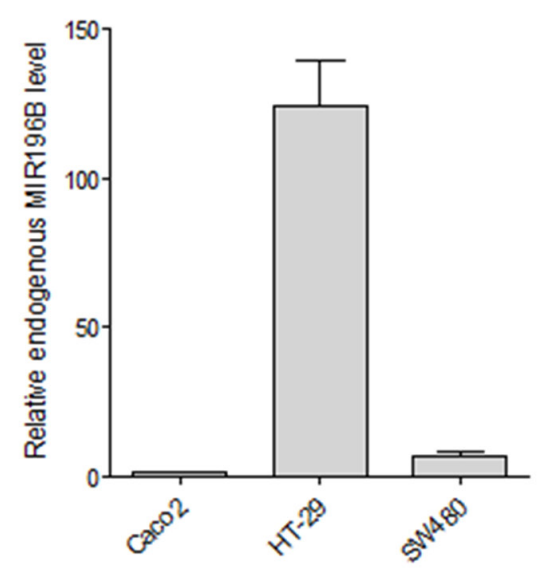

Figure 1: qRT-PCR analysis of miRNA expression in tissues and cells. (A) The expression of 11 miRNAs was validated using four colon cancer tissue samples and matched normal colon tissue samples. miRNAs levels were normalized with colon specific RNU48. Values are presented as the relative levels $(\Delta \Delta \mathrm{CT})$ of the miRNAs in colon cancer tissues. $P<0.01$ for all miRNAs by paired t-test. (B) MIR196B expression in 6 pairs of colorectal cancer tissue samples and adjacent normal colorectal tissue samples. Values are presented as the fold-change in tumor tissue relative to normal tissue. P1, P2, P5, and P6 indicate patients with colon cancer. P3 and P4 indicate patients with rectal cancer. $P<0.05$ for MIR196B by paired t-test. (C) The relative endogenous MIR196B expression levels in three colorectal cancer cell lines. Values are presented as the fold-change in HT29 or SW480 cells relative to Caco2 cells. $P<0.01$ for MIR196B by t-test. Data represent the mean \pm S.D. of three independent experiments, each carried out in duplicate. 
FAS-mediated active caspases after FAS monoclonal antibodie $(\mathrm{mAb})$ treatment in SW480 cells. FAS, CASP8, and CASP3 expression in SW480 cells was up-regulated (1.21, 1.72 and 1.39 fold, respectively) by FAS $\mathrm{mAb}$ treatment (Fig. 3B). The up-regulation of these proteins was significantly attenuated by MIR196B (Fig. 3B). We also assessed the expression of FasL, NF- $\mathrm{B}$ (p65), and ERK2 in FAS mAb-treated SW480 cells (Fig. 3C).
Whereas FasL expression was up-regulated (1.23 fold) by FAS mAb treatment, NF- $\kappa \mathrm{B}$ and ERK2 expression was slightly down-regulated ( 0.9 and 0.72 fold, respectively) by FAS mAb treatment (Fig. 3C). The up-regulation of FasL expression was attenuated by MIR196B transfection. Interestingly, NF- $\mathrm{B}$ and ERK2 levels were upregulated (1.54 and 1.41 fold, respectively) by MIR196B transfection (Fig. 3C).
A

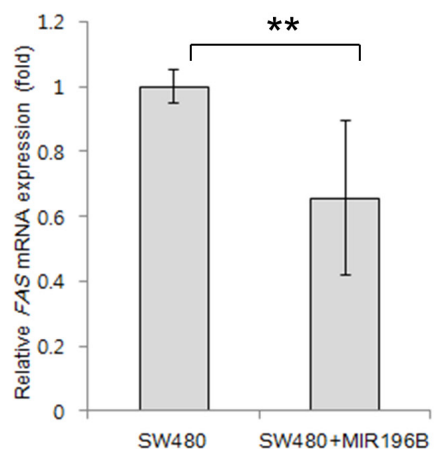

B

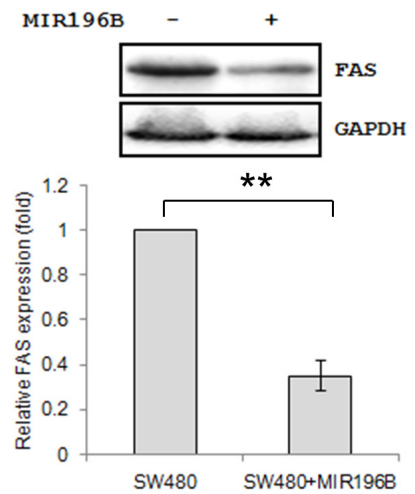

C

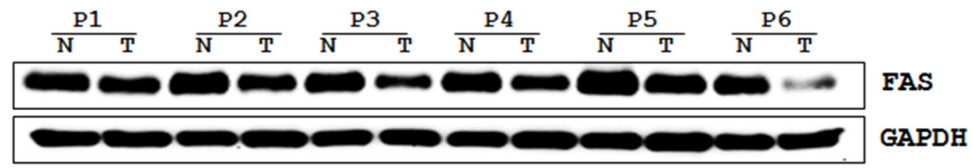

D

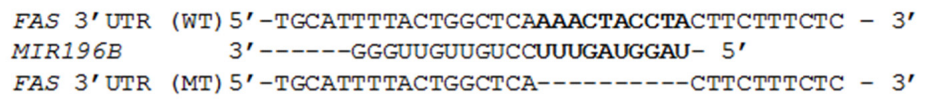

E

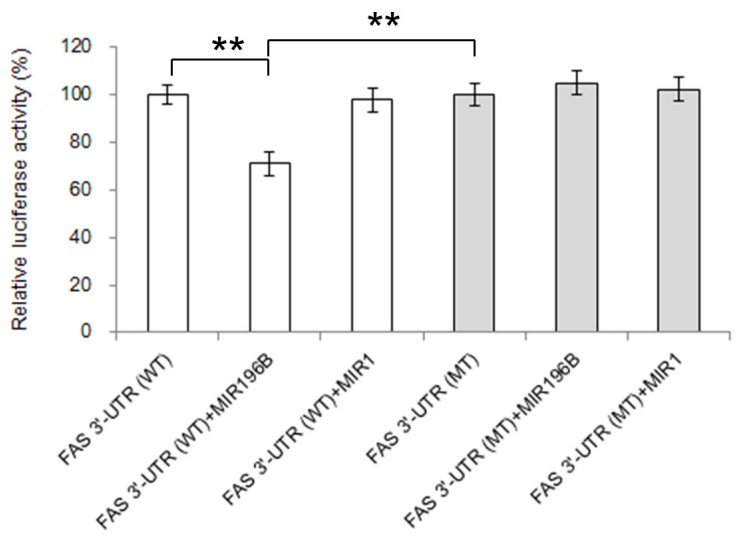

Figure 2: FAS is a direct target gene of MIR196B. (A) qRT-PCR analysis of FAS expression in SW480 cells. SW480 cells were mock-transfected or transfected with pre-MIR196B. Cells were harvested $48 \mathrm{~h}$ after transfection. Total RNA was extracted and used for qRT-PCR. Values are presented as the fold-change in MIR196B-overexpressing cells relative to non-transfected cells. The experiment was performed in duplicate and repeated five times. (B) FAS protein levels in MIR196B-overexpressing cells and non-transfected cells. Protein was extracted $72 \mathrm{~h}$ after transfection for western blot analysis. (C) FAS expression in 6 pairs of colon cancer tissue samples and adjacent normal colon tissue samples. All samples are from patients with colon cancer. (D) Sequence alignment of the wild-type (WT) and mutated (MT) MIR196B target site in the 3'-UTR of FAS. A human FAS 3' UTR containing the wild-type and mutant MIR196B binding sequence was cloned downstream of the luciferase reporter gene. (E) A luciferase reporter plasmid containing the WT or MT FAS 3' UTR was cotransfected into SW480 cells with pre-MIR1 as a negative control or pre-MIR196B. Luciferase activity was determined using the dual luciferase assay. Results are shown as the relative firefly luciferase activity normalized to Renilla luciferase activity. Data assessed from three independent experiments and the $P$ values were calculated by $t$-test $(* P<0.05$; $* * P<0.01)$. 
Table 2: MIR196B target genes

\begin{tabular}{|c|c|c|c|c|}
\hline Gene symbol & Accession & Gene name & $\begin{array}{c}\text { Chromosome } \\
\text { location }\end{array}$ & Functions \\
\hline HOXB7 & NM_004502.3 & homeobox B7 & $17 \mathrm{q} 21.3$ & transcription factor \\
\hline $\mathrm{HOXC8}$ & NM_022658.3 & homeobox $\mathrm{C} 8$ & $12 \mathrm{q} 13.3$ & transcription factor \\
\hline HOXA9 & NM_152739.3 & homeobox A9 & $7 \mathrm{p} 15.2$ & transcription factor \\
\hline GLTP & NM_016433.3 & glycolipid transfer protein & $12 \mathrm{q} 24.11$ & transfer/carrier protein \\
\hline LAMTOR5 & NM_006402.2 & $\begin{array}{l}\text { late endosomal adaptor, MAPK and } \\
\text { MTOR activator } 5\end{array}$ & $1 \mathrm{p} 13.3$ & regulate $\mathrm{HBx}$ activity \\
\hline ANXA1 & NM 000700.1 & annexin A1 & $9 q 21.13$ & transfer/carrier protein \\
\hline APEX2 & NM_014481.2 & APEX nuclease 2 & $\mathrm{Xp} 11.21$ & nucleic acid binding \\
\hline FAS & NM_152877.1 & cell surface death receptor & $10 \mathrm{q} 24.1$ & signal transduction \\
\hline HAND1 & NM_004821.1 & $\begin{array}{c}\text { heart and neural crest derivatives } \\
\text { expressed } 1\end{array}$ & $5 q 33$ & transcription factor \\
\hline HOXA5 & NM_019102.2 & $\begin{array}{c}\text { homeobox A5 } \\
\end{array}$ & $7 \mathrm{p} 15.2$ & transcription factor \\
\hline HOXB6 & NM_018952.4 & homeobox B6 & $17 \mathrm{q} 21.3$ & transcription factor \\
\hline KCNIP3 & NM_013434.4 & $\begin{array}{c}\mathrm{Kv} \text { channel interacting protein } 3, \\
\text { calsenilin }\end{array}$ & $2 q 21.1$ & calcium binding protein \\
\hline LRRC49 & NM_017691.2 & leucine rich repeat containing 49 & $15 \mathrm{q} 23$ & - \\
\hline MED10 & NM_032286.2 & mediator complex subunit 10 & $5 \mathrm{p} 15.31$ & transcription factor \\
\hline MRPL39 & NM_017446.3 & $\begin{array}{lll}\text { mitochondrial } & \text { ribosomal protein } \\
& \text { L39 }\end{array}$ & $21 \mathrm{q} 21.3$ & synthase and synthetase \\
\hline SPRED1 & NM_152594.1 & $\begin{array}{c}\text { sprouty-related, } \begin{array}{c}\text { EVH1 domain } \\
\text { containing } 1\end{array} \\
\end{array}$ & $15 q 14$ & receptor \\
\hline TGFB1I1 & NM_015927.3 & $\begin{array}{l}\text { transforming growth factor beta } 1 \\
\text { induced transcript } 1\end{array}$ & $16 \mathrm{p} 11.2$ & transcription cofactor \\
\hline VSNL1 & NM_003385.4 & visinin-like 1 & $2 \mathrm{p} 24.3$ & calcium binding protein \\
\hline
\end{tabular}

The target genes were identified by comparing mRNAs down-regulated in MIR196B-overexpressing cells with target genes predicted by public bioinformatics tools (TargetScan (http://www.targetscan.org) and miRWalk (http:// www.umm.uni-heidelberg.de/apps/zmf/mirwalk/index.html).

\section{MIR196B down-regulates FAS-mediated molecules in HT29 cells}

The endogenous MIR196B level in HT29 cells was higher than that in SW480 cells (Fig. 1C). Our results suggested that anti-MIR196B transfection would upregulate FAS expression in HT29 cells. We investigated the expression of FAS and FAS-mediated caspases in MIR196B- or anti-MIR196B-transfected HT29 cells. As expected, the expression of FAS (1.3 fold), active CASP8 (1.08 fold), and active CASP3 (1.56 fold) was up-regulated by anti-MIR196B transfection (Fig. 4A). As observed in SW480 cells, FAS, CASP8, and CASP3 levels in HT29 cells were markedly down-regulated $(0.57$, 0.77 and 0.65 fold, respectively) by MIR196B transfection (Fig. 4A). Interestingly, the pattern of active CASP9 expression after MIR196B and anti-MIR196B transfection in HT29 cells differed from the pattern in SW480 cells. CASP9 expression levels were almost unchanged by MIR196B or anti-MIR196B transfection in SW480 cells (Fig. 3A), whereas CASP9 expression was remarkably up-regulated ( 1.56 fold) and down-regulated ( 0.7 fold) in HT29 cells by anti-MIR196B and MIR196B transfection, respectively (Fig. 4A). We also determined the expression of FasL in HT29 cells. FasL expression was markedly down-regulated (0.61 fold) by MIR196B in HT29 cells. However, whereas FAS expression was up-regulated by anti-MIR196B transfection in HT29 cells, FasL expression (0.98 fold) did not change (Fig. 4A).

\section{Effect of MIRI96B on Caspase 3/7 activities}

We profiled and quantified, whether MIR196B can influence the activation of caspase 3/7 activities in SW480 and HT29 cells. As shown in Supplementary Figure 1D, MIR196B overexpression decreased caspase-3/7 activities in SW480 cells $(0.80$ fold change, $p<0.01)$ and HT29 cells $(0.89$ fold change, $\mathrm{p}<0.05)$, compared to the mock condition, respectively.

\section{Effect of MIR196B or anti-MIR196B transfection on apoptosis in colorectal cancer cells}

We analyzed apoptosis in MIR196B- or antiMIR196B transfected colorectal cancer cell lines. As shown in Fig. 4B, apoptosis in SW480 cells increased $(P$ $<0.05)$ after anti-MIR196B transfection. The increase $(P$ 
$<0.01)$ in apoptosis was also observed in anti-MIR196Btransfected HT29 cells (Fig. 4C upper panel). The number of apoptotic cells among anti-MIR196B-transfected HT29 cells was much higher than among mock-treated cells. Although the ratio was low, the number of apoptotic cells decreased $(P<0.05)$ after MIR196B transfection in SW480 and HT29 cells (Fig. 4B and Fig. 4C). When FAS $\mathrm{mAb}$ was added to SW480 cells, apoptosis increased (Fig. 4B lower panel). The increased apoptotic cell ratio was not changed by MIR196B or anti-MIR196B transfection in FAS mAb-treated SW480 cells.
Anti-MIR196B overexpression increased 5-FUinduced apoptosis in HT29 cells

We examined the $\mathrm{IC}_{50}$ values of 5-FU using MTT assay after the cells were exposed to 5-FU. The $\mathrm{IC}_{50}$ of 5 -FU for HT29 was $4 \pm 0.02 \mu \mathrm{M} / \mathrm{ml}$. We investigated whether anti-MIR196B or MIR196B played a functional role in 5-FU-induced apoptosis. The apoptosis rate was significantly increased $(P<0.01)$ in HT29 cells after 5-FU treatment compared to untreated control cells (Fig. 4C, lower panel). Anti-MIR196B or MIR196B overexpression increased $(P<0.01)$ or decreased $(P<0.05)$ the 5-FU-
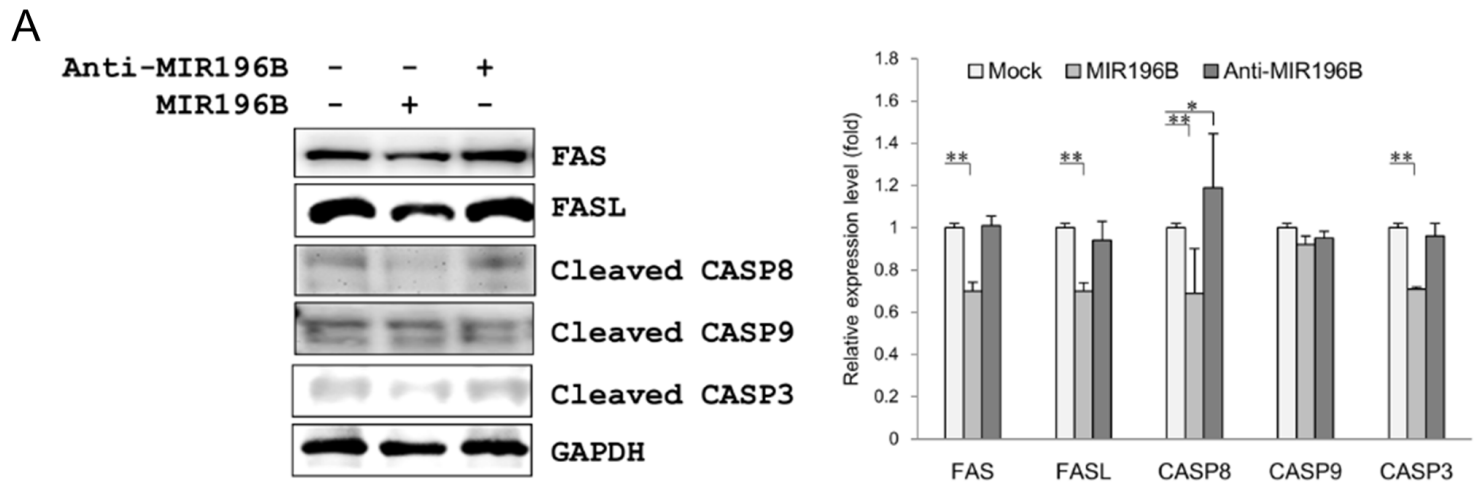

B
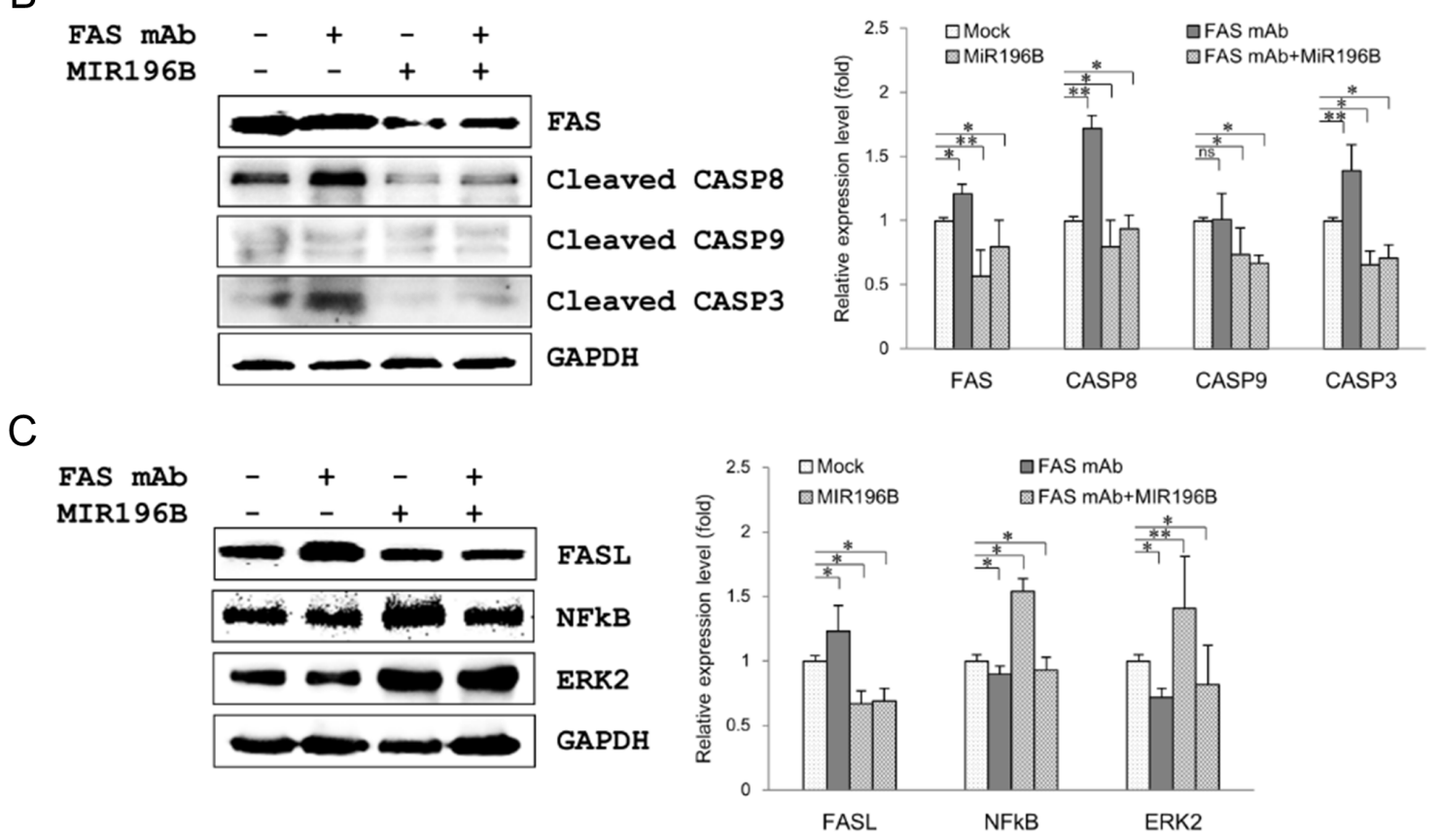

Figure 3: MIR196B regulates FAS-mediated apoptosis in SW480 cells. (A) Western blot analyses of FAS, FasL, and FASmediated molecules in MIR196B- or anti-MIR196B-transfected SW480 cells. Proteins were extracted $72 \mathrm{~h}$ after transfection for western blot analysis. The data shown were normalized to GAPDH levels and are presented as fold-change in MIR196B- or anti-MIR196Btransfected cells relative to mock-transfected cells. The experiment was repeated four times. (B) Western blot analyses of FAS and FASmediated molecules in FAS mAb-treated or MIR196B-transfected SW480 cells. (C) Western blot analyses of FasL, NF-kB, and ERK2 in FAS mAb-treated or MIR196B-transfected SW480 cells. Protein was extracted $72 \mathrm{~h}$ after MIR196B transfection or FAS mAb treatment or $48 \mathrm{~h}$ after MIR196B transfection into cells cultured with FAS mAb for $24 \mathrm{~h}$. The data shown were normalized to GAPDH levels and are presented as the fold-change in MIR196B-transfected or FAS mAb-treated cells relative to mock-treated cells. Data assessed from four independent experiments and the $P$ values were calculated by $t$-test $(* P<0.05 ; * * P<0.01$; ns $=$ not significant $)$. 
induced apoptosis in HT29 cells, compared to the cells treated with 5-FU, respectively. The result showed that the overexpression of anti-MIR196B up-regulated the 5-FU induced apoptosis in colon cancer cells (Fig. 4C, lower panel).

\section{DISCUSSION}

miRNAs have been implicated as important regulators of gene expression in a variety of biological processes in various diseases, as well as in various diseases as tumor suppressor genes or oncogenes [9-11]. Using colon cancer tissues and normal colon tissues, we identified 31 colon cancer-associated miRNAs by miRNA microarray analysis and validated the results by qRT-PCR (Table 1 and Fig. 1A). We selected MIR196B for further study. MIR196B was up-regulated in human colorectal cancer tissue (Fig. 1B). Most functional studies of MIR196B have reported that the miRNA is deregulated in various leukemias [18-24]. We used mRNA microarray analysis and bioinformatics tools to identify MIR196B target genes in colorectal cancer.

FAS is a death domain-containing member of the TNF receptor superfamily [25]. FAS plays a central role in the physiological regulation of apoptosis, and it has been implicated in the pathogenesis of various malignancies [26]. In human cancer, tumor cells tend to downregulate FAS expression to avoid FAS-mediated apoptosis signaling [27, 28], particularly in metastatic human colorectal cancer $[29,30]$. We showed that MIR196B regulates FAS and the FAS-mediated apoptosis pathway by directly downregulating FAS expression (Figs. 2-4). These results suggest that MIR196B is a regulator of FASmediated apoptosis in colorectal cancer.

Apoptosis is a distinct cell death program that is primarily triggered by the intrinsic or extrinsic apoptosis pathway. The intrinsic pathway is activated by cytotoxic stress and is characterized by the release of cytochrome c from the mitochondria, resulting in the activation of downstream CASP9 and CASP3 [31]. The extrinsic pathway is activated by the binding of FasL to FAS, leading to CASP8 and CASP3 activation, which initiates
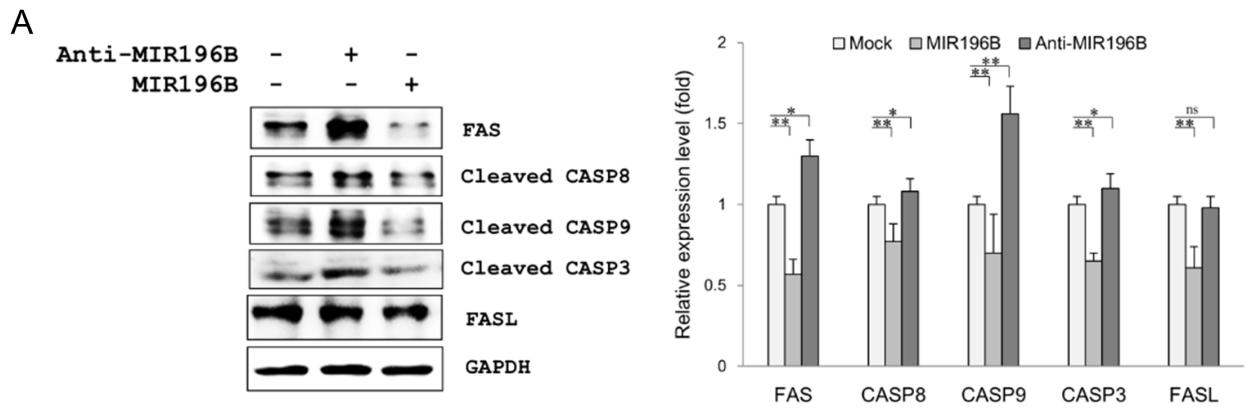

B
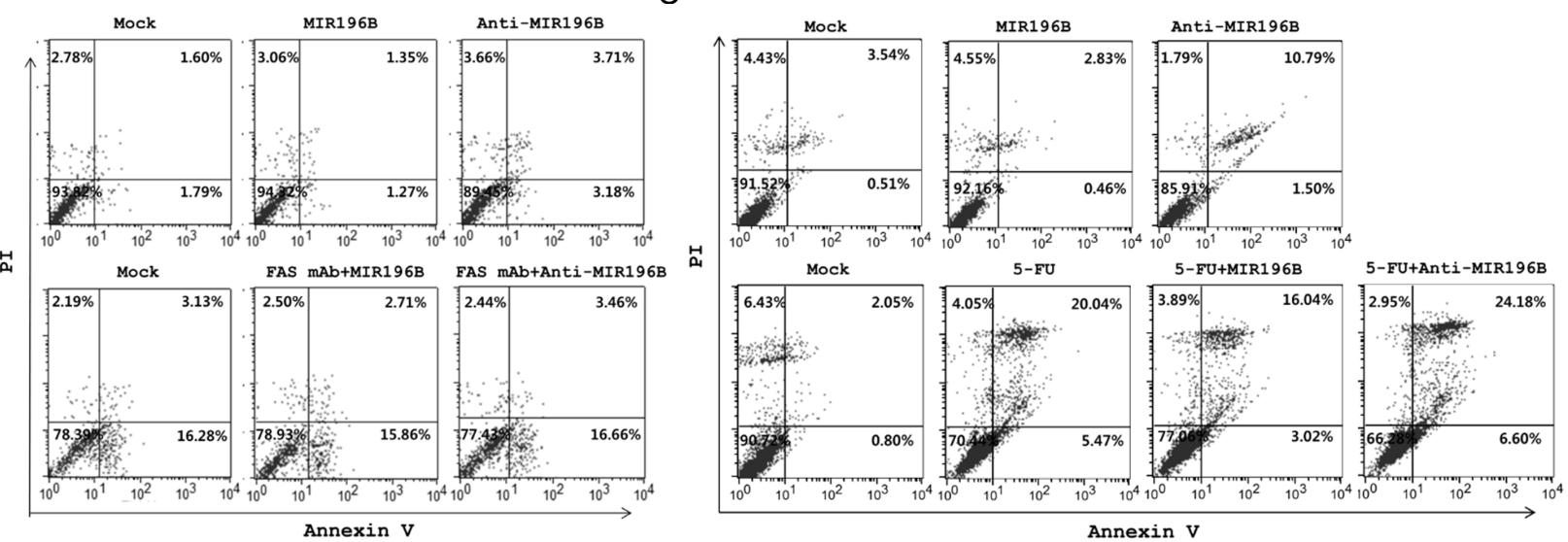

Figure 4: MIR196B regulates FAS-mediated apoptosis in SW480 or HT29 cells. (A) Western blot analyses of FAS, FasL, and FAS-mediated molecules in MIR196B- or anti-MIR196B-transfected HT29 cells. Experiments were repeated four times and the $P$ values were calculated by $t$-test $(* P<0.05$; ** $P<0.01$; ns = not significant). (B) Flow cytometry analysis of FAS-mediated apoptosis in MIR196B- or anti-MIR196B-transfected SW480 cells. Cells were cultured in the absence (upper panel) or presence (lower panel) of FAS $\mathrm{mAb}$, stained with annexin V and PI, and analyzed by flow cytometry. The number in each box indicates the percentage of annexin V-and/ or PI-positive cells. (C) Flow cytometry analysis of apoptosis in MIR196B- or anti-MIR196B-transfected HT29 cells. Cells were cultured in the absence (upper panel) or presence (lower panel) of 5-FU. Cells were collected $72 \mathrm{~h}$ after transfection, stained with annexin V and PI, and analyzed by flow cytometry. The number in each box indicates the percentage of annexin V-and/or PI-positive cells. Experiments were repeated three times with duplicates. 
multiple pro-apoptotic processes [32]. In this study, we showed that FAS, CASP8, and CASP3 expression in SW480 and HT29 cells was markedly down-regulated by MIR196B overexpression and that anti-MIR196B overexpression restored their expression (Fig. 3A and 4A). As we expected, the effect of anti-MIR196B was stronger in HT29 cells than in SW480 cells. The latter expressed lower levels of MIR196B than HT29 cells (Fig. 1C). Extrinsic pathway molecules were down-regulated by MIR196B in both cell lines, whereas the intrinsic pathway molecule CASP9 was regulated in a cell-type specific manner (Figs. 3A and B and 4A). Many miRNAs partially regulate target gene expression in specific cells or tissues. FAS was constitutively expressed at high levels in normal human colon tissues, but its expression was often reduced in human colorectal cancer tissues. Our results suggest that the reduction in FAS level was due to increased MIR196B expression in colorectal cancer. The reduction in FAS was not sufficient to induce apoptosis in colorectal cancer cells.

FAS is a critical component of the FAS-mediated apoptosis pathway and thus an attractive target in cancer therapy. FAS mAbs could act as effective anticancer agents by activating FAS-mediated apoptosis, as shown in the lower panel of Fig. 4B. However, FAS mAb induces extensive apoptosis in hepatocytes, resulting in lethal liver damage [33,34], thus limiting the clinical use of FAS mAbs for systemic anticancer chemotherapy. As shown in Fig. 4B and Fig. 4C, MIR196B overexpression decreased the apoptotic cell ratio in SW480 and HT29 cells, whereas anti-MIR196B transfection increased apoptosis. These results indicate that MIR196B regulates the FAS-mediated apoptosis pathway by modulating FAS expression. Therefore, our results suggest that antiMIR196B is a candidate material for anticancer therapy in human colorectal cancer. We also carried out the effect of MIR196B and anti-MIR196B with an anti-cancer agent 5-FU in HT29 cells (Fig. 4C, lower panel). These results indicated that anti-MIR196B increased the sensitivity of 5-FU, suggest that MIR196B may contribute to chemoresistance in colon cancer.

FasL expression was only observed in colorectal carcinomas, but FAS was constitutively expressed at high levels in normal human colon tissues. However, FAS expression is often reduced in metastatic human colorectal carcinoma [29, 35]. FasL expression in tumor cells has been hypothesized as a "FAS counterattack," by which tumor cells evade immune destruction by inducing FasLmediated apoptosis in tumor-infiltrating lymphocytes [36]. However, this concept is legitimately debated [37, 38]. Interestingly, our results showed that FasL expression in SW480 and HT29 cells was markedly down-regulated by MIR196B overexpression (Fig. 3A and 4A). Furthermore, the up-regulation of FasL induced by FAS mAb treatment was attenuated by anti-MIR196B overexpression in SW480 cells (Fig. 3C). The FasL expression patterns in response to MIR196B and anti-MIR196B overexpression were similar to the FAS expression patterns (Fig. 3A and $\mathrm{C}$ and Fig. 4A). These results emphasizes that MIR196B regulates FasL expression by an unknown mechanism, although we did not establish this in the present study.

We measured NF- $\mathrm{BB}$ and ERK2 expression after MIR196B overexpression (Fig. 3C). Whereas FAS and FasL expression was down-regulated, NF- $\kappa \mathrm{B}$ and ERK2 expression was up-regulated by MIR196B (Fig. 3C). Consequently, we assessed whether MIR196B regulated cell viability via the NF- $\kappa \mathrm{B}$ or ERK2 pathway in SW480 and HT29 cells (Fig. S2). However, we did not find any evidence in association between MIR196B expression and cell proliferation in colorectal cancer cells.

In summary, we identified 31 miRNAs that were significantly up-regulated or down-regulated in colorectal cancer tissues. We investigated one of them, MIR196B, and identified 18 putative MIR196B target genes. FAS was verified as MIR196B targets in this study. MIR196B expression was up-regulated in colorectal cancer tissue, whereas FAS expression was down-regulated. MIR196B directly repressed FAS expression in colorectal cells. In contrast, anti-MIR196B up-regulated FAS expression and increased apoptosis in colorectal cancer cell lines. Our results suggest that up-regulated MIR196B modulates apoptosis in colorectal cancer cells by partially repressing FAS expression and that anti-MIR196B could be useful as an anti-cancer drug in colorectal cancer.

\section{MATERIALS AND METHODS}

\section{Patients and tissue samples}

The tissue samples used in this study were provided by the Biobank of Wonkwang University Hospital, a member of the National Biobank of Korea. With approval from the institutional review board and informed consent, we obtained colon cancer tissue from 14 colon cancer patients ( 7 males and 7 females) and rectal cancer tissue from 2 rectal cancer patients ( 2 males). The mean ages of the colon cancer patients and rectal cancer patients were 65.6 years and 72 years, respectively. Four colon cancer tissue samples and matched normal colon tissue samples ( 2 males and 2 females) were used for miRNA microarray analysis. Three separate colon cancer tissue samples and matched normal colon tissue samples ( 1 male and 2 female) were used to validate the miRNA microarray results. In addition, one separate colon cancer tissue sample with matched normal colon tissue sample and two rectal cancer tissue samples with matched normal rectal tissue samples were used to assess MIR196B expression. Six separate colon cancer tissue samples and matched normal colon tissue samples ( 4 males and 2 females) were used to analyze FAS protein expression. 


\section{Cell culture}

The human colorectal cancer cell lines SW480, HT29, and Caco2 were obtained from Korea Cell Line Bank. SW480 and HT29 cells were cultured in RPMI 1640 (HyClone, Logan, UT, USA) supplemented with $10 \% \mathrm{FBS}$ in $5 \% \mathrm{CO}_{2}$ at $37^{\circ} \mathrm{C}$ in a humidified atmosphere. $\mathrm{Caco} 2$ cells were cultured in Alpha-MEM (HyClone) supplemented with $20 \% \mathrm{FBS}$ in $5 \% \mathrm{CO}_{2}$ at $37^{\circ} \mathrm{C}$ in a humidified atmosphere.

\section{miRNA expression profiling}

Total RNA (100 ng) was hybridized to an Agilent Human microRNA Microarray (Agilent Technologies, Santa Clara, CA, USA). MicroRNA was labeled, hybridized, and washed following Agilent's instructions. Images of hybridized microarrays were acquired with a DNA microarray scanner (Agilent Technologies), and the microarray images were analyzed with Feature Extraction software (Agilent Technologies). The standard of statistical significance was the corrected ratio of the hybridization signal intensity in colon tumor tissue to the hybridization signal intensity in normal tissue.

\section{RNA extraction and quantitative RT-PCR}

Total RNA was isolated with TRIzol reagent (Invitrogen, Carlsbad, CA, USA) according to the manufacturer's protocol. After digestion with DNase and cleanup, RNA samples were quantified, aliquoted, and stored at $-80^{\circ} \mathrm{C}$. Total RNA isolated from tissue samples and/or cultured cells were used as a template to synthesize cDNA for quantitative RT-PCR (qRT-PCR) analysis in a StepOne Real-time PCR system (Applied Biosystems, Foster City, CA, USA).

The differential miRNA expression patterns were validated with the TaqMan qRT-PCR assay (Applied Biosystems) or the NCode VILO miRNA cDNA Synthesis kit for qRT-PCR and EXPRESS SYBR GreenER miRNA qRT-PCR kit (Invitrogen). qRT-PCR with SYBR Green dye (Applied Biosystems) was used to assess mRNA expression. RNU48 (for TaqMan qRT-PCR) or 5.8S (for SYBR qRT-PCR) and GAPDH were used as endogenous controls for qRT-PCR of miRNA and mRNA, respectively. Each sample was run in triplicate.

\section{MIR196B transfection}

SW480 and HT29 cells $\left(3 \times 10^{5}\right)$ or Caco 2 cells $\left(1.5 \times 10^{5}\right)$ were plated in 6-well or $10-\mathrm{cm}$ culture plates and cultured as described above. MIR196B (hsa-miR196b, Pre-miR miRNA Precursor AM17100, Product ID: PM12946) or anti-MIR196B (anti-hsa-miR-196b,
Anti-miR miRNA Inhibitor AM17000, Product ID: AM12946) were commercially synthesized (Ambion, Austin, TX, USA) and transfected at $50 \mathrm{nmol} / \mathrm{L}$ using Lipofectamine RNAiMAX (Invitrogen) or siPORTTM NeoFX ${ }^{\mathrm{TM}}$ transfection agent (Ambion) according to the manufacturers' recommendations. Cells were harvested 24-48 $\mathrm{h}$ (for miRNA and mRNA) or 48-72 h (for protein) after transfection for functional assays, flow cytometry assays, or RNA/protein extraction.

\section{mRNA expression profiling of MIR196B target genes}

SW480 and Caco2 cells were transfected with MIR196B or MIR1 as a control. Total RNA was isolated $48 \mathrm{~h}$ after transfection, amplified, and purified using the Illumina TotalPrep RNA Amplification Kit (Ambion) according to the manufacturer's instructions to yield biotinylated complementary RNA (cRNA). Labeled cRNA samples (750 ng) were hybridized to a HumanHT-12 v4 Expression BeadChip array (Illumina, Inc., San Diego, CA, USA) for $16-18 \mathrm{~h}$ at $58^{\circ} \mathrm{C}$. The array signal was detected using Amersham fluorolink streptavidin-Cy3 (GE Healthcare Bio-Sciences, Little Chalfont, UK), following the instructions in the BeadChip array manual. Arrays were scanned with an Illumina BeadArray Reader according to the manufacturer's instructions. Array data was exported, processed, and analyzed using Illumina BeadStudio v3.1.3 (Gene Expression Module v3.3.8). Array data were filtered for a detection p-value $<0.05$ (similar to signal-to-noise) in at least 50\% samples.

\section{MIR196B target prediction by bioinformatics methods}

The miRNA targets were predicted using the computer-aided algorithms TargetScan (http://www. targetscan.org) and miRWalk (http://www.umm.uniheidelberg.de/apps/zmf/mirwalk/index.html).

\section{Plasmid constructions and luciferase assays}

Wild-type or mutant fragments of the $3^{\prime}$ untranslated region (UTR) of FAS containing the predicted binding site for MIR196B, were amplified by PCR using the primer set shown in Table S2. The PCR product was cloned into the pmirGLO Dual-Luciferase miRNA Target Expression Vector (Promega, Madison, WI, USA).

For reporter assays, cells $\left(5 \times 10^{4} /\right.$ well $)$ were seeded in 24-well plates and co-transfected with wildtype or mutant FAS constructs (500 ng/well) or with $50 \mathrm{nM}$ MIR196B or MIR-1 (negative control) using Lipofectamine 2000 (Invitrogen Life Technologies) and siPORT ${ }^{\mathrm{TM}} N e o F X^{\mathrm{TM}}$ Transfection Agent (Ambion), 
respectively, according to manufacturers' instructions. Firefly and Renilla luminescence was measured $24 \mathrm{~h}$ after transfection using the Dual-Glo Luciferase Assay System (Promega) according to the manufacturer's instructions. Non-transfected cells were used for background subtraction, and the ratio of firefly reporter luminescence to Renilla reporter luminescence (control) was calculated. All experiments were performed in triplicate and repeated at least three times.

\section{Antibodies and western blot analysis}

SW480 or HT29 cells $\left(2 \times 10^{5}\right.$ cells/well $)$ were seeded in 6-well plates and incubated for $72 \mathrm{~h}$. Whole cell lysates were prepared by incubation in RIPA buffer supplemented with a protease inhibitor mixture for 30 min at $4^{\circ} \mathrm{C}$. Protein was collected by centrifugation at $12,000 \mathrm{rpm}$ for $30 \mathrm{~min}$ at $4^{\circ} \mathrm{C}$. Equal amounts of protein (50 $\mu \mathrm{g}$; determined by the Bradford assay) were boiled in Laemmli buffer, subjected to $12.0 \%$ or $15.0 \%$ SDS-PAGE, and transferred to PVDF membranes. The membranes were incubated with blocking buffer [5\% BSA in TBS containing $0.1 \%$ Tween-20 (TBS-T)] for $2 \mathrm{~h}$ at room temperature. Membranes were then incubated overnight at $4^{\circ} \mathrm{C}$ with primary antibodies to FAS (G-9), FasL (C-178), ERK1/2 (H-72), NF-кB p65 (Santa Cruz Biotechnology), caspase-9 (human specific; \#9502), caspase-8 (1C12; \#9746) (Cell Signaling Technology, Boston, MA, USA) and caspase-3 (Enzo Life Sciences) and washed three times for 10 min per wash with TBS-T. Membranes were incubated with HRP-conjugated rabbit or mouse IgG secondary antibodies for $1 \mathrm{~h}$ at room temperature. After washing three times for $10 \mathrm{~min}$ in TBS-T, protein was detected with ECL solution (Millipore Corporation, Billerica, MA, USA) and a FluorChem E System (Cell Biosciences, Santa Clara, CA, USA). After protein detection, some membranes were stripped with stripping buffer for $1 \mathrm{~h}$ at room temperature and re-probed with antibody to GAPDH (0411; Santa Cruz Biotechnology), used as a loading control. Protein expression was evaluated using ImageJ software (version 1.44; http:// rsbweb.nih.gov/ij/index.html).

\section{Caspase 3/7 assay}

Caspase 3 and 7 activation assays were performed using the Caspase-Glo 3/7 Assay Kit (Promega) according to the manufacturer's instructions. Briefly, the cells were seeded in 96-well plates at a density of $2 \times 10^{4}$ cells/well and transfected with MIR196B or control. After 48h of transfection, Caspase-Glo 3/7 Reagent $(100 \mu 1)$ was added to each well and mixed gently using a plate shaker at 500 rpm for $30 \mathrm{sec}$. The plate was then incubated at room temperature for $2 \mathrm{~h}$ in the dark. The luminescence of each sample was measured by using a GloMax ${ }^{\circledR}-$ Multi+
Detection System (Promega). The data were analyzed with GraphPad Prism 5 (GraphPad Software Inc., San Diego, USA).

\section{Viability (MTT) assay}

The effect of MIR196B and antisense MIR196B (anti-MIR196B) on cell viability was evaluated with the MTT (3-(4,5-dimethylthiazol-2-yl)-2-5diphenyltetrazoliumbromide) assay (M2128; SigmaAldrich, St. Louis, MO, USA). SW480 or HT-29 cells (2 $\times 10^{4}$ cells/ well) were transfected with $50 \mathrm{nM}$ MIR196B or anti-MIR196B in a 96-well plate and grown for 72 h. The medium was carefully removed, and cells were washed twice with $1 \times$ PBS. MTT solution $(5 \mathrm{mg} / \mathrm{ml})$, dissolved in culture medium at a final concentration of $0.5 \mathrm{mg} / \mathrm{ml}$, was added to each well. The plates were wrapped in aluminum foil and incubated for another $3 \mathrm{~h}$ at $37^{\circ} \mathrm{C}$. The medium was removed, and $100 \mu \mathrm{l}$ of DMSO (Duchefa Biochemie, Haarlem, The Netherlands) was added to solubilize the MTT tetrazolium crystals. Finally, the solution was agitated with a pipette until no change in color was observed, and the optical density was read at $560 \mathrm{~nm}(\mathrm{OD} 560)$ using a GloMax ${ }^{\circledR}-$ Multi+ Detection System (Promega). The percentage of viable cells was estimated by comparison with the untreated controls. At least three independent experiments were performed. The $\mathrm{IC}_{50}(50 \%$ inhibitory concentration) values of 5-FU (F6627, Sigma-Aldrich Co., Missouri, USA) was assessed by MTT assay. The molar concentration required for $50 \%$ inhibition of cell viability $\left(\mathrm{IC}_{50}\right)$ was calculated. In the following experiments, the concentration of 5-FU used were equal to the $\mathrm{IC}_{50}$ and duration of 5-FU treatment was $72 \mathrm{~h}$ in HT29 cells.

\section{Apoptosis analysis (flow cytometry)}

Apoptosis was analyzed by flow cytometry with the Annexin V-FITC Apoptosis Detection Kit according to the manufacturer's instructions (Sigma-Aldrich). Briefly, SW480 or HT29 cells $\left(1 \times 10^{5}\right.$ /well or $3 \times 10^{5} /$ well) were seeded in 6-well plates and collected $72 \mathrm{~h}$ after transfection with MIR196B or anti-MIR196B or control. SW480 cells $\left(3 \times 10^{5} /\right.$ well $)$ were also seeded in 6-well plates, cultured $24 \mathrm{~h}$ with FAS mAb (anti-FAS/ CD95 monoclonal antibody; MBL, Nagoya, Japan), and collected $48 \mathrm{~h}$ after transfection with MIR196B, antiMIR196B, or control. 5-FU $(4 \mu \mathrm{M} / \mathrm{ml})$ was added $10 \mathrm{~h}$ after transfection and the cells were incubated for $72 \mathrm{~h}$. The cells were trypsinized, washed in cold PBS, and resuspended in $100 \mu 11 \times$ binding buffer. To estimate the apoptotic cell number, cells were double-stained with annexin $\mathrm{V}$ and propidium iodide (PI) solution, incubated for $20 \mathrm{~min}$ on ice, and resuspended in $1 \times \operatorname{PBS}(300 \mu \mathrm{l})$. Samples were then analyzed using a FACSCalibur (Becton 
Dickinson, USA) and CellQuest analysis software (Becton Dickinson). For each sample, 10,000 cells were analyzed.

\section{Statistical analysis}

Each experiment was repeated at least three times with consistent results. All of the data were represented as mean \pm standard deviation (SD). Statistical differences were analyzed by GraphPad prism 5.0 statistical software (GraphPad Software Inc., San Diego, USA) or Student's t-test, and p-values of less than $0.05(\mathrm{p}<0.05)$ were regarded as statistically significant.

\section{ACKNOWLEDGMENTS}

The biospecimens for this study were provided by the Biobank of Wonkwang University Hospital, a member of the National Biobank of Korea, which is supported by the Ministry of Health and Welfare. This research was supported by the Basic Science Research Program through the National Research Foundation of Korea (NRF), funded by the Ministry of Science, ICT, and Future Planning (NRF-2012R1A2A2A01010517).

\section{Statement of author contributions}

The authors have made the following declarations about their contributions: Conceived and designed the experiments: CSC. Performed the experiments: MJS, AKJ and KIH. Analyzed the data: MHB, YKJ and CSC. Contributed reagents/materials/analysis tools: PWC, SGS, $\mathrm{CSC}$ and KHS. Wrote or revision the paper: MJS, AKJ, KHS and CSC.

\section{Conflict of Interest}

The authors have no competing interest to declare

\section{REFERENCES}

1. Parkin DM, Bray F, Ferlay J and Pisani P. Global cancer statistics, 2002. CA: A Cancer Journal for Clinicians. 2005; 55(2):74-108.

2. Swan J, Breen N, Coates RJ, Rimer BK and Lee NC. Progress in cancer screening practices in the United States: results from the 2000 National Health Interview Survey. Cancer. 2003; 97:1528-1540.

3. Giovannucci E. Modifiable risk factors for colon cancer. Gastroenterology Clinics of North America. 2002; 31(4):925-943.

4. Larsson SC, Orsini N, and Wolk A. Diabetes mellitus and risk of colorectal cancer: a meta-analysis. Journal of the National Cancer Institute. 2005; 97(22):1679-1687.
5. Itzkowitz $\mathrm{SH}$ and Yio X. Inflammation and cancer IV. Colorectal cancer in inflammatory bowel disease: the role of inflammation. American Journal of Physiology Gastrointestinal and Liver Physiology. 2004; 287(1):G7-17.

6. Esquela-Kerscher A and Slack FJ. Oncomirs - microRNAs with a role in cancer. Nature Reviews Cancer. 2006; 6(4):259-269.

7. Hammond SM. MicroRNAs as oncogenes. Current Opinion in Genetics and Development. 2006; 16(1):4-9.

8. Ambros V. The functions of animal microRNAs. Nature. 2004; 431:350-355.

9. Bartel DP. MicroRNAs: genomics, biogenesis, mechanism, and function. Cell. 2004; 116(2):281-297.

10. Garzon R, Fabbri M, Cimmino A, Calin GA and Corce CM. MicroRNA expression and function in cancer. Trends in Molecular Medicine. 2006; 12:580-587.

11. Lu J, Getz G, Miska EA, Alverez-Saavendra E, Lamb J, Peck D, Ebert BL, Mak RH, Ferrando AA, Downing JR, Jacks T, Horvitz HR Golub TR. MicroRNA expression profiles classify human cancers. Nature. 2005; 435(7043):834-838.

12. Yanaihara N, Caplen N, Bowman E, Seike M, Kumamoto K, Yi M, Stephens RM, Okamoto A, Yokota J, Tanaka T, Calin GA, Liu CG, Croce CM et al. Unique microRNA molecular profiles in lungcancer diagnosis and prognosis. Cancer Cell. 2006; 9(3):189-198.

13. Lowery AJ, Miller N, McNeill RE and Kerin MJ. MicroRNAs as prognostic indicators and therapeutic targets: potential effect on breast cancer management. Clinical Cancer Research. 2008; 14(2):360-365.

14. Schetter AJ, Leung SY, Sohn JJ, Zanetti KA, Bowman ED, Yanaihara N, Yuen ST, Chan TL, Kwong DL, Au GK, Liu CG, Calin GA, Croce CM et al. MicroRNA expression profiles associated with prognosis and therapeutic outcome in colon adenocarcinoma. JAMA. 2008; 299(4):425-436.

15. Yekta S, Shih IH, and Bartel DP. MicroRNAdirected cleavage of HOXB8 mRNA. Science. 2004; 304(5670):594-596.

16. Tanzer A, Amemiya CT, Kim CB, and Stadler PF. Evolution of microRNAs located within Hox gene clusters. Journal of Experimental Zoology Part B: Molecular and Developmental Evolution. 2005; 304(1):75-85.

17. Chen C, Zhang Y, Zhang L, Weakley SM and Yao Q. MicroRNA-196: critical roles and clinical applications in development and cancer. Journal of Cellular and Molecular Medicine. 2011; 15(1):14-23

18. Bhatia S, Kaul D and Varma N. Potential tumor suppressive function of miR-196b in B-cell lineage acute lymphoblastic leukemia. Molecular and Cellular Biochemistry. 2010; 340(1-2):97-106.

19. Bhatia S, Kaul D and Varma N. Functional genomics of tumor suppressor miR-196b in T-cell acute lymphoblastic leukemia. Molecular and Cellular Biochemistry. 2011; 346(1-2):103-116. 
20. Coskun E, von der Heide EK, Schlee C, Kühnl A, Gökbuget N, Hoelzer D, Hofmann WK, Thiel E and Baldus CD. The role of microRNA-196a and microRNA 196b as ERG regulators in acute myeloid leukemia and acute T-lymphoblastic leukemia. Leukemia Research. 2011; 35(2):208-213

21. Li Z, Huang H, Chen P, He M, Li Y, Arnovitz S, Jiang J, He C, Hyjek E, Zhang J, Zhang Z, Elkahlon A, Cao D et al. miR-196b directly targets both HOXA9/MEIS1 oncogenes and FAS tumour suppressor in MLL-rearranged leukaemia. Nature Communication. 2012; 3:688. doi: 10.1038/ ncomms1681.

22. Liu Y, Zheng W, Song Y, Ma W and Yin H. Low expression of miR-196b enhances the expression of BCR-ABL1 and HOXA9 oncogenes in chronic myeloid leukemogenesis. PLoS One. 2013; 8(7):e68442. doi: 10.1371.

23. Popovic R, Riesbeck LE, Velu CS, Chaubey A, Zhang J, Achille NJ, Erfurth FE, Eaton K, Lu J, Grimes HL, Chen J, Rowley JD and Zeleznik-Le NJ. Regulation of mir-196b by MLL and its overexpression by MLL fusions contributes to immortalization. Blood. 2009; 113(14):3314-22.

24. Schotte D, Lange-Turenhout EA, Stumpel DJ, Stam RW, Buijs-Gladdines JG, Meijerink JP, Pieters R and Den Boer ML. Expression of miR-196b is not exclusively MLLdriven but is especially linked to activation of HOXA genes in pediatric acute lymphoblastic leukemia. Haematologica. 2010; 95(10):1675-1682.

25. Itoh N, Yonehara S, Ishii A, Yonehara M, Mizushima S, Sameshima M, Hase A, Seto Y and Nagata S. The polypeptide encoded by the cDNA for human cell surface antigen Fas can mediate apoptosis. Cell. 1991; 66(2):233243

26. Nagata S. Fas and Fas ligand: a death factor and its receptor. Advances in Immunology. 1994; 57: 129-144.

27. Hanahan D and Weinberg RA. The hallmarks of cancer. Cell. 2000; 100:57-70.

28. Stewart TJ and Abrams SI. How tumours escape mass destruction. Oncogene. 2008; 27(45):5894-5903.

29. Moller P, Koretz K, Leithauser F, Brüderlein S, Henne C, Quentmeier A and Krammer PH. Expression of APO-1 (CD95), a member of the NGF/TNF receptor superfamily, in normal and neoplastic colon epithelium. International Journal of Cancer. 1994; 57(3):371-377.

30. Strater J, Hinz U, Hasel C, Bhanot U, Mechtersheimer G, Lehnert T and Moller P. Impaired CD95 expression predisposes for recurrence in curatively resected colon carcinoma: clinical evidence for immunoselection and CD95L mediated control of minimal residual disease. Gut. 2005; 54(5):661-665.

31. Adams JM and Cory S. The Bcl-2 apoptotic switch in cancer development and therapy. Oncogene. 2007; 26:1324-1337.

32. Wajant $H$. The Fas signaling pathway: more than a paradigm. Science. 2002; 31:296(5573):1635-1636.

33. Nagata S. Apoptosis by death factor. Cell. 1997; 88:355-
365.

34. Ogasawara J, Watanabe-Fukunaga R, Adachi $M$, Matsuzawa A, Kasugai T, Kitamura Y, Itoh N, Suda T and Nagata S. Lethal effect of the anti-Fas antibody in mice. Nature. 1993; 364(6440):806-809.

35. Krammer PH. CD95's deadly mission in the immune system. Nature. 2000; 407(6805):789-795.

36. O'Connell J, Bennett MW, O’Sullivan GC, Roche D, Kelly J, Collins JK and Shanahan F. Fas ligand expression in primary colon adenocarcinomas: Evidence that the Fas counterattack is a prevalent mechanism of immune evasion in human colon cancer. Journal of Pathology. 1998; 186(3):240-246.

37. Restifo NP. Countering the 'counterattack' hypothesis. Nature Medicine. 2001; 7(3):259.

38. Ryan AE, Shanahan F, O'Connell J and Houston AM. Fas ligand promotes tumor immune evasion of colon cancer in vivo. Cell Cycle. 2006; 5(3):246-249. 